\title{
First Results of the Analysis of the Wolf-Rayet Star WR6
}

\author{
Alex C. Gormaz-Matamala ${ }^{1}$ Anthony Hervé2, André-Nicolas Chené ${ }^{3}$, \\ Michel Curé ${ }^{4}$ and Ronald Mennickent ${ }^{1}$ \\ ${ }^{1}$ Departamento de Astronomía, Universidad de Concepción, Casilla 160-C, Concepción, Chile \\ email: agormaz@astro-udec.cl \\ ${ }^{2}$ CNRS \& Université Montpellier II, Place Eugene Bataillon, F-34095 Montpellier, France \\ ${ }^{3}$ Gemini Observatory, Hawaii, USA. \\ ${ }^{4}$ Instituto de Física y Astronomía, Universidad de Valparaíso. Casilla 5030, Valparaíso, Chile
}

\begin{abstract}
We present the first results of our analysis of the famous variable star, WR6 (HD50896). Using IUE ultraviolet data and an ESPaDOnS spectropolarimetric survey of this star, we plan to determine possible variation of the stellar and wind parameters during the different phases using the radiative transfer code CMFGEN. After the detection of parameter's modifications as a function of the phase, we will analyse deeper the origin of these variability (for example, CIRs?). In the present poster we show the first results of our analysis of the variability and the first step of the stellar parameter determination of the average spectrum of this star.
\end{abstract}

Keywords. Atmospheres, Mass Loss, Wolf-Rayet.

\section{Observational Data and Computational Tools}

For optical data, we use ESPaDOnS spectropolarimeter spectra provided by de la Chevrotière et al. 2013, whereas for UV data we use IUE spectra from MAST database. For the models, we use the code CMFGEN (Hillier et al. 2001).

\section{First Results}

\subsection{Variability}

Using the formalism of the Temporal Variation Spectrum (TVS: Fullerton et al. 1996; Chené 2007; St-Louis et al. 2009), together with defining one significant variability level $\sigma_{0}^{2} \chi_{N}^{2}-1(99 \%)$ from the standardised dispersion of our data, we can confirm WR6 line profile variability, as the TVS of the four studied lines are way above this threshold.

\subsection{Stellar Wind Parameter Determinations}

The parameters found and the current best model fit are shown in Table 1 and Figure 1. The terminal velocity was calculated using the CIV 1548 P-Cygni profile: our value coincides with that found by Hamann et al. (2006). For temperature, we used the HeII 5411/He I 5875 ratio (Smith et al. 1996).

\subsection{Further Work}

We must check the effects of different $\beta$ values yet. Also, is necessary to continue testing different abundances (specially carbon and nitrogen) for getting a best fit in those lines. 
Table 1. Stellar wind parameters and abundances of our current best fit model (Figure 1).

\begin{tabular}{|cc|cc|}
$\mid$ Parameter & Value & Parameter & Value | \\
\hline$T_{\text {eff }}[\mathrm{kK}]$ & 60 & {$\left[\mathrm{He} / \mathrm{He}_{\odot}\right]$} & 0.525 \\
$\dot{M}\left[M_{\odot} \mathrm{yr}^{-1}\right]$ & $1.9 \times 10^{-5}$ & {$\left[\mathrm{C} / \mathrm{C}_{\odot}\right]$} & -0.860 \\
$v_{\infty}\left[\mathrm{km} \mathrm{s}^{-1}\right]$ & 1700 & {$\left[\mathrm{~N} / \mathrm{N}_{\odot}\right]$} & 1.437 \\
{$\left[\mathrm{H} / \mathrm{H}_{\odot}\right]$} & -1.523 & {$\left[\mathrm{O} / \mathrm{O}_{\odot}\right]$} & 0.068 \\
\hline
\end{tabular}
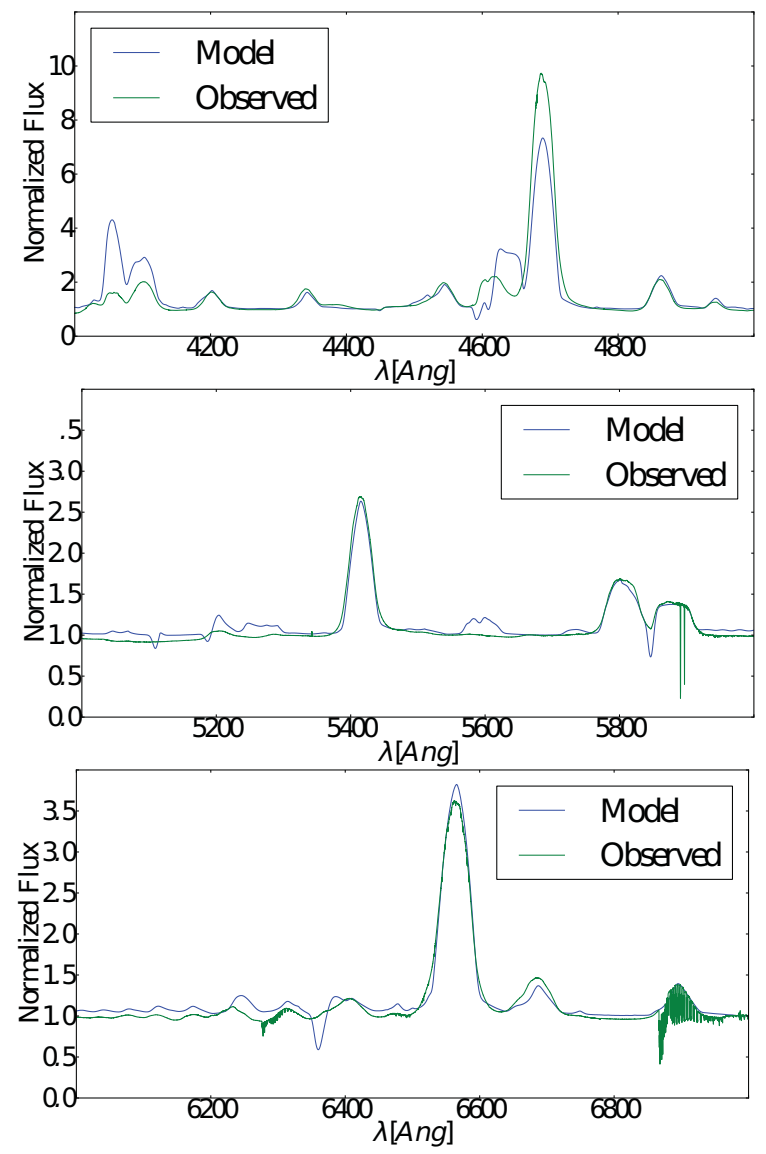

Figure 1. Model compared with the global averaged spectrum.

\section{References}

Chené, A.-N. 2007, Ph.D. thesis, Université de Montreal (Canada)

de la Chevrotière et al., A. 2013, ApJ 764, p.171

Fullerton et al., A. W. 1996, ApJS 103, p.475

Hamann et al., W. R. 2006, A\&A 457, 1015

Hillier et al., J. 2001, Spectroscopic Challenges of Photoionized Plasmas, ASP Conference Series. 247, p. 343

Smith et al., L. F. 1996, MNRAS 281, 163

St-Louis et al., N. 2009, ApJ 698, 1951 